\title{
Sequential and simultaneous synthesis problem solving: A comparison of students' gaze transitions
}

\author{
Bashirah Ibrahim $\oplus^{1}$ and Lin Ding $\oplus^{2, *}$ \\ ${ }^{1}$ Bahrain Teachers College, University of Bahrain, Zallaq, 1054, Bahrain \\ ${ }^{2}$ Department of Teaching and Learning, The Ohio State University, Columbus, Ohio 43210, USA
}

(Received 6 September 2020; accepted 10 March 2021; published 16 April 2021)

\begin{abstract}
This study examines students' visual behaviors when they tackle two types of synthesis problems, sequential and simultaneous problems. Sequential synthesis tasks can be solved by applying pertinent concepts consecutively, whereas simultaneous synthesis tasks require concurrent application of multiple concepts. Twenty-two students from an introductory calculus-based physics course participated in the study. We used an eye-tracker to record the students' eye movements when they silently reflected on how to solve the problems and subsequently when they talked aloud their problem-solving strategies. We found that the students made more gaze transitions between text and diagram for the simultaneous problems than for the sequential ones. However, they spent more time looking at the diagram and making within-diagram eye transitions in the sequential tasks than in the simultaneous tasks. Further, most students invoked two concepts to solve the sequential tasks but only one for the simultaneous tasks. These findings indicate that the students made less effort to link text and diagram in solving sequential problems but frequently attempted to integrate information within each diagram. The pattern for the simultaneous problems appeared to be reversed. Our results suggest that different types of synthesis (i.e., sequential and simultaneous) may differentially influence the ways students handle tasks. As suggested by the eye-tracker data and confirmed by the participants' verbal explanations, students tend to divide the situation in sequential problems into subtasks but treat simultaneous problems as a single-step task.
\end{abstract}

DOI: 10.1103/PhysRevPhysEducRes.17.010126

\section{INTRODUCTION}

Although research on physics problem solving abounds $[1,2]$, it has been mostly limited to single-concept problems [3-8] involving oversimplified situations with little bearing on the real world [9]. Such problems require students to apply what is being currently taught to devise a solution [10] thereby promoting algorithmic use of concepts. Also, they are mainly quantitative end-of-chapter exercises and only train students to manipulate equations in a rote manner. Overall, single-concept problems may not support students in qualitative analysis of the tasks.

\section{A. Sequential and simultaneous synthesis problems}

In this study, we use synthesis problems that require students to integrate two or more distinct concepts

\footnotetext{
*Corresponding author. ding.65@osu.edu

Published by the American Physical Society under the terms of the Creative Commons Attribution 4.0 International license. Further distribution of this work must maintain attribution to the author(s) and the published article's title, journal citation, and DOI.
}

typically taught at different time points and in different chapters [10-12]. We distinguish two types of synthesis problems, namely, sequential and simultaneous synthesis problems. Sequential synthesis problems (see Fig. 1) involve a series of chronologically occurring events and require consecutive applications of corresponding principles [13,14]. For example, Q1 in Fig. 1 involves two consecutive events: a block is first propelled by a spring and then undergoes a trajectory to land on an inclined surface. In this case, two fundamental principles, conservation of energy and projectile motion, need to be applied for the two events respectively. As another example, Q3 in Fig. 1 depicts a sequence of two phenomena, a rollercoaster descending on a track and then going onto a circular hill. Correspondingly, it requires application of two pertinent concepts, respectively, conservation of energy and Newton's second law.

Simultaneous problems (see Fig. 2) contain a series of phenomena that require concurrent applications of multiple principles $[13,14]$. For example, in Q2 (see Fig. 2) a bullet hitting a rod results in both translational and rotational motions of the system. In Q4 (see Fig. 2), after a clay blob hits a disk and sticks to it, the whole clay blob-disk system begins to rotate and translate simultaneously. In both cases, two concepts are needed to solve the problem; they are 
Q1: An apparatus uses a spring launcher to fire a block (mass, $M=2 \mathrm{~kg}$ ) over a ridge. The spring (spring constant, $\mathrm{k}=3000 \mathrm{~N} / \mathrm{m}$ ) is compressed by $0.1 \mathrm{~m}$ and releases the block from its equilibrium position, located at a distance $L=0.5 \mathrm{~m}$ from the crest. If the angles shown are $\theta=35^{\circ}$ and $\phi=25^{\circ}$, what is the horizontal distance $R$ from the crest to where the block hits the slope on the other side?

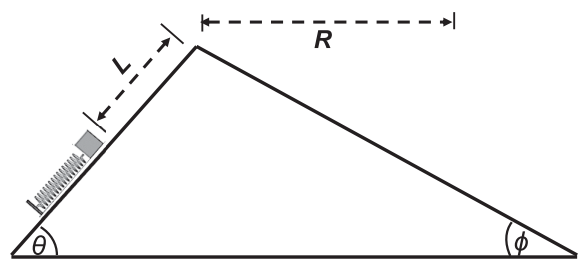

Q3: You are tasked with designing a rollercoaster. As part of the design, the track gradually descends until it comes to a small semi-circular hill of height $R=80 \mathrm{~m}$. You know that the speed of the rollercoaster cart is $v=19 \mathrm{~m} / \mathrm{s}$ when the cart of mass $M=100 \mathrm{~kg}$ is $20 \mathrm{~m}$ above the height of the oncoming hill. What is the normal force acting on the cart when it is at the top of the hill? Ignore friction.

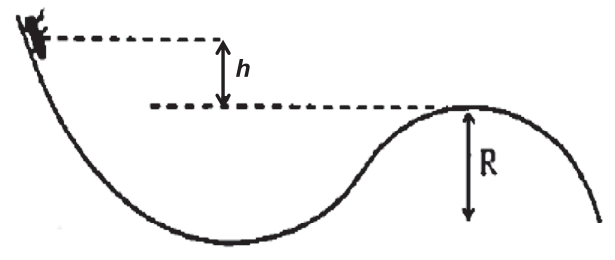

FIG. 1. Two sequential synthesis problems.

Q2: A rod of mass $m=1 \mathrm{~kg}$ and length $L=10 \mathrm{~cm}$ rests freely on a frictionless surface. A bullet of mass $M=0.02 \mathrm{~kg}$ is fired with a velocity of $v_{0}=$ $300 \mathrm{~m} / \mathrm{s}$ such that it hits the edge of the rod. The bullet rips through the rod, emerging in the same direction, with a speed of $v=100 \mathrm{~m} / \mathrm{s}$. What is the total kinetic energy of the rod after the collision? (Moment of inertia of the rod is $I=$ $1 / 12 \mathrm{ML}^{2}$ ).

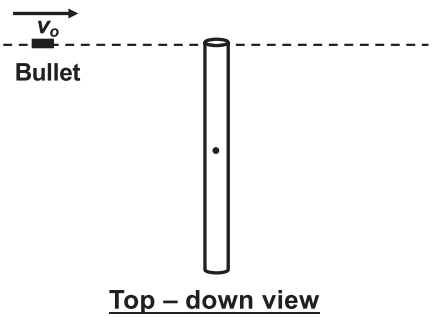

Q4: Tom throws a clay blob of mass $0.02 \mathrm{~kg}$ at a puck placed on a horizontal frictionless air table. The clay moves at $50 \mathrm{~m} / \mathrm{s}$ in a direction tangent to the rim of the puck just before sticking onto its outer rim. Initially stationary, the system of puck plus clay after impact begins moving on the surface. The puck is a uniform disk of mass $1 \mathrm{~kg}$ and radius $R=0.75 \mathrm{~m}$. What is the final total kinetic energy of the puck plus clay system? [Moment of inertia of uniform disks $=1 / 2 M R^{2}$ ]

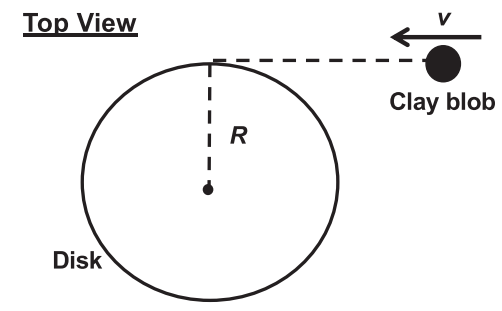

FIG. 2. Two simultaneous synthesis problems.

conservation of linear momentum and conservation of angular momentum.

We use synthesis problems for the following considerations. They may help to channel students' attention away from novicelike approaches to problem solving [10]. Synthesis problems cannot be easily solved by manipulating equations; instead they emphasize conceptual analysis. When handling tasks involving multiple phenomena, students need to perform qualitative sensemaking to identify appropriate concepts, understand connections thereof, and reflect on suitable solution strategies. In terms of using equations, synthesis problems may also support students' understandings of the link between multiple equations they generate before quantitative calculations $[13,14]$. However, there is a lack of research that directly investigates how students solve multiconcept problems. Previous studies have mainly explored the effectiveness of using interventions, involving multiconcept tasks, to develop expertlike problemsolving approaches among students [9,15-17].

\section{B. Purpose of study and eye-tracking methodology}

Our work is novel in that we classify synthesis problems into two types, namely, sequential and simultaneous tasks. Each possesses unique features (as described above) that may differentially influence students' problem-solving patterns. Based on this hypothesis, we aim at gaining insights into how students unpack the two types of synthesis tasks by looking specifically into the ways that 
the students connect text and diagram as well as the various components within each diagram to tackle the problems. We gathered evidence from the students' visual data and verbal responses to achieve this goal.

We use an eye tracker to record students' visual attention and gaze transitions during synthesis problem solving. Visual attention relates directly to the location at which a person's eyes are fixated [18] and gaze transitions refer to shifts in visual attention between various regions [19]. Prior research using eye tracker to explore students' problem solving across various disciplines has mainly used single-concept problems. These studies have often relied on fixation-based metrics such as the number and duration of fixations to compare the visual behaviors between novice students and experts (successful and unsuccessful problem solvers) [20-27]. In physics education research (PER), few studies have incorporated measures of eye movements such as the number of gaze transitions [4]. Our work differs from prior eye-tracking studies in PER in at least two ways. First, we use synthesis problems to investigate how the different characteristics of sequential and simultaneous problems may influence students' visual behaviors. We are interested in how students make sense of a diagram and connect it to the text for the two types of synthesis. It is important to emphasize that it is not our intent to compare successful and unsuccessful problem solvers. Instead, we hope to explore how different synthesis types influence the ways students process information. Second, we examine both eye fixation and transition to conclude about students' visual behaviors. Here we look at (i) students' fixation duration on problem text and diagram, and (ii) the number of gaze transitions between problem text and diagram, and between different components of a diagram. To summarize, our research question is as follows: How do students' fixation durations and gaze transitions compare when they handle sequential and simultaneous synthesis problems?

Eye tracker allows us to gather direct evidence of students' visual attention [28,29]. Most importantly, it provides a measure of students' cognitive processes in real time $[18,30]$. Studies using eye-tracking methodology are based on the "eye-mind assumption" [31,32]. According to Just and Carpenter [31], to process the meaning of a word one needs to fixate on it. The fixation continues until the processing is over. Hence, the fixation duration is directly related to the processing of the word. Moreover, Irwin [32] suggested that cognitive processing occurs both during eye movements (such as gaze transitions) and when the eyes are fixated at a location. Research exploring students' problemsolving processes has provided evidence of the connection between visual attention and cognitive mechanisms $[20,27,33,34]$. Of particular interest are the studies examining students' gaze transitions to understand how text and mathematics or text and pictures are used for learning [4,28,35-38]. In their research exploring how students study worked-out examples in mechanics, Smith, Mestre, and Ross [4] interpreted gaze transitions between corresponding textual and mathematical information as students' combining information from both sources. Johnson and Mayer [30] additionally highlighted the role of cognition in gaze transitions. According to them, gaze transitions between text and diagram reflect cognitive attempts to integrate and process information between two representations to comprehend a task. They argued that for meaningful learning to occur students need to integrate the words and picture elements in their working memory. According to the cognitive theory of multimedia learning [39], when handling tasks with pictures and texts, students need to process both in the working memory. They also need to build a mental representation of the situation by organizing and combining picture and text with their prior knowledge from the long-term memory. Overall, more gaze transitions and fixation durations mean greater integrative processing of information and hence deeper learning [40,41].

In our study, a core requirement for solving synthesis problems is to link multiple events and concepts to make sense of the situations. By focusing on students' gaze transitions and fixation durations, we can study the extent to which they attempt to process and integrate information when tackling the two types of synthesis problems.

\section{METHOD}

\section{A. Synthesis problems and areas of interests (AOIs)}

The four problems used in this study (Figs. 1 and 2) were taken from previously published work [10,13, $14,42,43]$. These problems contain two types of areas of interests (AOIs), text (problem statement) and diagram. The latter was further divided into different regions, depicting entities and labels in the diagram. The AOIs in the diagram for the four synthesis problems are shown in the Appendix. Table I shows the number of AOIs in the diagram for the four problems. Overall, the difference in the number across the four synthesis tasks is small.

TABLE I. Number of AOIs in the diagram for the sequential and simultaneous synthesis tasks.

\begin{tabular}{llccc}
\hline \hline & \multicolumn{2}{l}{ Sequential tasks } & \multicolumn{2}{c}{ Simultaneous tasks } \\
\hline AOIs & Q1 & Q3 & Q2 & Q4 \\
Diagram & 10 & 11 & 9 & 11 \\
\hline \hline
\end{tabular}




\section{B. Administration of tasks}

Our sample comprises 22 students enrolled in an introductory calculus-based physics class. These students had not been extensively exposed to synthesis problems in their physics courses. At the time of the study they had learned the relevant topics in class.

Each student participated in a session that lasted for a maximum of one hour. Each session comprised a thinking phase and a talking phase, where we recorded the students' eye movements by using EyeLink-1000. During the thinking phase, the students were given a maximum of 10 min to silently think of each task and reflect on how they would tackle the problem. They moved to the next question once they reported that they understood the problem and could solve it. They repeated the process for each of the four tasks. On average, the students spent 6 min thinking silently on each problem. During the talking phase, the students saw the same four problems but were asked to verbalize their thoughts in response to the following prompts: (i) Tell me, in your words, what is going on in this situation. (ii) Tell me, how you will solve this problem. We are not looking for formulae that you will use but the general strategy that you will use to solve the problem. (iii) Is there anything else you want to add? Overall, the students spent a maximum of 6 min on each problem talking aloud their thoughts. Their verbal responses were audio taped and transcribed.

\section{Analysis}

For students' visual patterns in each task, we calculated the percentages of fixation durations on diagram and text. We also studied the number of gaze transitions between text and diagram, and between the different AOIs in diagram. We conducted a Kolmogorov-Smirnov test and a chi squared test on our datasets and found no

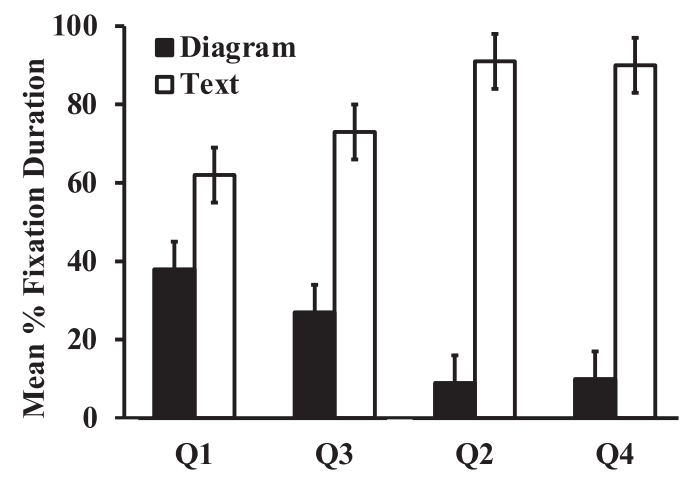

Sequential Tasks Simultaneous Tasks

(a) evidence for violation of normal distribution with equal variance (thinking phase, all $p>0.22$; talking phase, all $p>0.59$ ). Hence, we used the parametric statistics multiway repeated ANOVA to compare (i) the mean percentages of fixation durations on diagram and text, and (ii) the mean percentages of gaze transitions between text and diagram, and between the different AOIs in diagram.

Regarding the verbal data, we coded the number of concepts identified by the students for each task and the relevancy thereof. This information was gathered by considering whether the students explicitly or implicitly referred to the concept(s) while describing their strategies to solve each problem. The verbal responses were coded independently by two researchers. An interreliability rate of $87 \%$ was reached across the four tasks.

\section{RESULTS}

\section{A. Students' eye fixation duration}

We calculated the mean percentages of students' eye fixation durations on diagram and text, respectively, when they solved the two types of synthesis problems during both the thinking and talking phases (see Fig. 3). As seen, the students had a longer fixation duration on each of the sequential diagrams than they did on each simultaneous diagram. Conversely, the pattern for fixation on text is reversed. These results were found to be statistically significant [thinking phase: $F(3,84)=51.6, \quad p<0.001$; talking phase: $F(3,84)=$ $32.9, p<0.001$; Bonferroni pairwise comparisons error rate $\alpha<0.0083$.]

\section{B. Students' gaze transitions}

Figure 4 shows the mean percentages of gaze transitions within the diagram, and between text and diagram

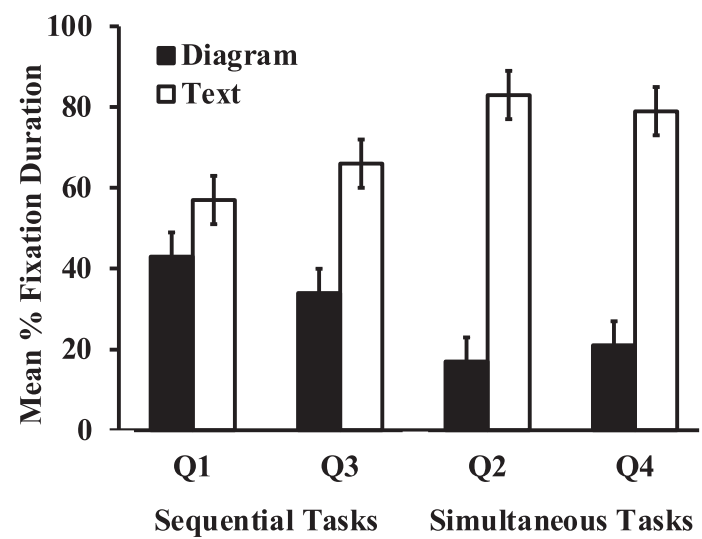

(b)

FIG. 3. Mean percentages fixation durations ( \pm standard error) on diagram and text for (a) the thinking phase and (b) the talking phase across the four problems. 


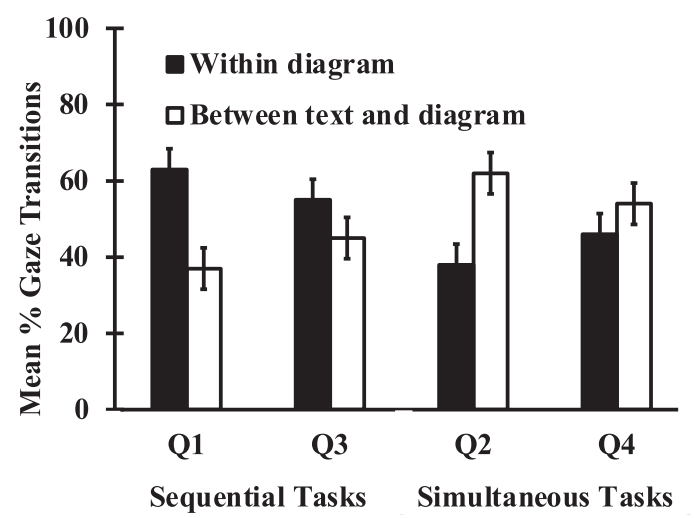

(a)

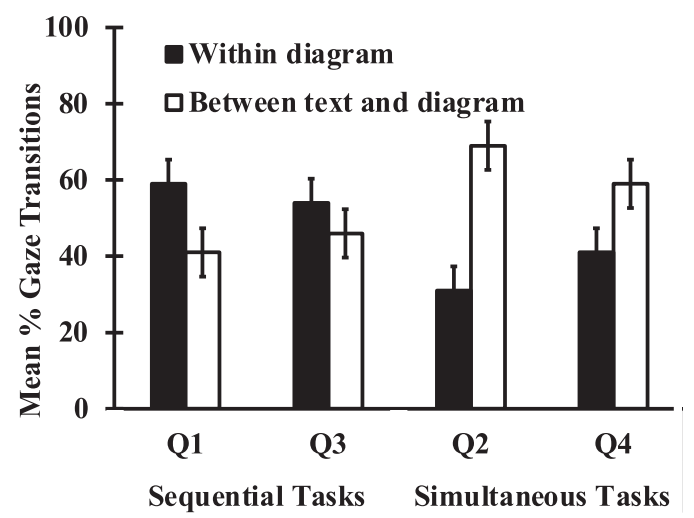

(b)

FIG. 4. Mean percentages of gaze transitions ( \pm standard error) within diagram, and between text and diagram for the (a) thinking phase and (b) talking phase.

for the two types of synthesis problems during both the thinking and talking phases. Overall, the students made more within-diagram gaze transitions for the sequential problems than for the simultaneous ones. Conversely, their text-diagram transitions in the sequential problems were less frequent than in the simultaneous problems. These results were statistically significant [thinking phase: $F(3,84)=18.7, \quad p<0.001 ;$ talking phase: $F(3,84)=26.7, p<0.001$, Bonferroni pairwise comparisons error rate $\alpha<0.0083]$.

\section{Students' verbal responses}

From the students' verbal responses, we identified the number and types of concepts they used to tackle the problems (see Table II). For the sequential synthesis tasks, most students identified two concepts, regardless of correctness (Q1: 95\%, Q3: 83\%). In contrast, for the simultaneous synthesis tasks, most students identified only one concept (Q2: 73\%, Q4: 69\%).

Typical responses for the sequential (Q1 and Q3) and simultaneous (Q2 and Q4) synthesis tasks are shown in Table III. For example, a student invoked two concepts to solve Q1, one irrelevant (Newton's second law) and one relevant (projectile motion). The student directly stated using projectile motion but only hinted at Newton's second law by referring to its corresponding mathematical equation. For Q3, one student identified two pertinent concepts. He explicitly mentioned conservation of energy but alluded to Newton's second law by identifying the forces acting on the rollercoaster and the presence of centripetal acceleration.

For simultaneous problems, most students only identified one concept. For example, a student explicitly identified conservation of mechanical energy (an irrelevant concept) for Q2. For Q4, a student explicitly invoked conservation of angular momentum (a relevant concept).

We also conducted an ad hoc analysis to see whether there is a difference in the mean percentage of withindiagram gaze transitions between those students who identified all the relevant concepts and those who did not. No significant difference was detected, and the results hold for both types of synthesis problems in the thinking phase (all $p>0.10$ ) and in the talking phase (all $p>0.14$ ). Details of the statistical results are shown in Table IV.

TABLE II. Number and types of concepts students identified for the sequential and simultaneous problems $(N=22)$.

\begin{tabular}{|c|c|c|c|c|c|}
\hline & & \multicolumn{2}{|c|}{ Sequential tasks } & \multicolumn{2}{|c|}{ Simultaneous tasks } \\
\hline \multicolumn{2}{|c|}{ Number and types of concepts } & Q1 & Q3 & Q2 & Q4 \\
\hline \multirow[t]{3}{*}{ Two concepts } & Both relevant & $68 \%$ & $64 \%$ & $27 \%$ & $27 \%$ \\
\hline & One relevant and one irrelevant & $22 \%$ & $14 \%$ & $0 \%$ & $4 \%$ \\
\hline & Both irrelevant & $5 \%$ & $5 \%$ & $0 \%$ & $0 \%$ \\
\hline \multirow[t]{2}{*}{ One concept } & Relevant & $5 \%$ & $0 \%$ & $23 \%$ & $14 \%$ \\
\hline & Irrelevant & $0 \%$ & $17 \%$ & $50 \%$ & $55 \%$ \\
\hline
\end{tabular}


TABLE III. Examples of interview responses.

\begin{tabular}{|c|c|}
\hline $\begin{array}{l}\text { Question } \\
\text { number }\end{array}$ & Interview excerpt \\
\hline Q1 & $\begin{array}{l}\text { For the [spring] force I'll multiply } 3000 \mathrm{~N} / \mathrm{m} \text { by } 0.1 \mathrm{~m} \text {. I have the angle of projection. I would take the vertical and } \\
\text { horizontal components of that force and divide each force component by the mass to get acceleration of the block. I } \\
\text { will then use projectile motion, horizontal and vertical motion equations to get the time [...]. }\end{array}$ \\
\hline Q3 & $\begin{array}{l}\text { You'll use conservation of energy to find the velocity of the rollercoaster at the top of the hill. Then, you do a force } \\
\text { diagram. The forces acting on it [the rollercoaster] are the normal force and gravity. Then, you set the forces equal to } \\
m v^{2} / R[\ldots] \text { to find the normal force. }\end{array}$ \\
\hline Q2 & $\begin{array}{l}\text { The final kinetic energy is the same as the initial since there is no loss in energy in the system. I need to calculate the } \\
\text { change in energy of the bullet as it passes through the rod. Its initial velocity is } 300[\mathrm{~m} / \mathrm{s}] \text { and final velocity is } \\
100[\mathrm{~m} / \mathrm{s}] . \text { I have the mass. I can find the initial and final kinetic energy }[\ldots] . \text { By conservation of energy, the total } \\
\text { before and after of the system should be the same. }\end{array}$ \\
\hline Q4 & $\begin{array}{l}\text { We know the velocity of the clay blob, its mass, the radius, and we know the amount of initial angular momentum, } \\
M V R \text {. When the clay blob impacts the disk, there is } M V R \text { and angular momentum of the disk. The moment of inertia } \\
\text { for the clay blob is } M R^{2} \text { and moment of inertia of disk is } 1 / 2 M R^{2} \omega \text {. The equation will be } \\
M V R=1 / 2 M R^{2} \omega+M R^{2} \text {. We can find } \omega \text {. To find the total kinetic energy of the system, it is the rotational kinetic } \\
\text { energy } 1 / 2 I \omega^{2} \text {. }\end{array}$ \\
\hline
\end{tabular}

TABLE IV. Statistical results from one-way ANOVA comparing within-diagram gaze transitions between students who identified all the correct concepts and those who did not.

\begin{tabular}{lllcc}
\hline \hline & & $F$ statistic & $p$ value \\
\hline Thinking phase & \multirow{2}{*}{ Sequential tasks } & Q1 & 2.82 & 0.10 \\
& & Q3 & 0.01 & 0.91 \\
& \multirow{4}{*}{ Simultaneous tasks } & Q2 & 0.17 & 0.68 \\
& & Q4 & 2.76 & 0.12 \\
Talking phase & \multirow{4}{*}{ Sequential tasks } & Q1 & 0.16 & 0.69 \\
& & Q3 & 1.27 & 0.27 \\
& \multirow{4}{*}{ Simultaneous tasks } & Q2 & 0.10 & 0.76 \\
& & Q4 & 2.33 & 0.14 \\
\hline \hline
\end{tabular}

\section{CONCLUSIONS AND DISCUSSIONS}

Our findings show that there are noticeable differences in how students handle the two types of synthesis problems. For simultaneous synthesis tasks, the students fixated heavily on the text and made frequent gaze transitions between text and diagram. From the vantage point of the eye-mind assumption [31] as well as the cognitive theory of multimedia learning [39] and the work by Johnson and Mayer [30], this suggests that perhaps the diagrams alone in the simultaneous problems are not self-explanatory enough for the students to make sense of the situation; therefore, they had to repeatedly rely on both text and diagram to integrate information in order to understand the tasks. However, the students made fewer gaze transitions within the diagram for each simultaneous task, indicating less cognitive effort being made to link information between different components in a diagram. A possible reason for these outcomes is that perhaps the students did not realize each static diagram implied multiple events, and as a result they treated it as a single event. Our findings from student verbal data indeed showed that they mostly invoked one concept to solve the simultaneous tasks.

In contrast, our results show that the students spent more time looking at the diagram for the sequential tasks than for the simultaneous ones. Moreover, they made fewer gaze transitions between text and diagram in the sequential tasks compared to those in the simultaneous ones. Drawing again on the cognitive theory of multimedia learning [39] and the work by Johnson and Mayer [30], we can infer that the students made greater cognitive attempts to process diagrams than to perform text-diagram integration. Overall, these outcomes suggest that the sequential diagrams may be fairly self-explanatory. Hence, the students did not need to frequently refer back to the text to understand the situation. However, their eyes shifted greatly among various parts of each diagram, revealing their mental effort to dovetail different pieces into a whole. In other words, the students might have recognized multiple events in each sequential problem and were actively seeking to piece them together for a solution. This seemed to be consistent with our findings from the students' verbal responses showing that they mostly identified two concepts to solve the sequential tasks.

The differences in students' visual reactions to sequential and simultaneous tasks as evidenced in our study are largely associated with the distinct characteristics of the two types of synthesis. As mentioned above, we did not detect any meaningful difference in the visual patterns between those who correctly identified relevant concepts and those who did not. Therefore, our findings about the influences of synthesis types on students' visual behaviors were independent of their success in solving the problems. Instead, the differences in student visual patterns seemed largely to originate from the different synthesis types. In each of the simultaneous synthesis problems, multiple events take place concurrently such that it is necessary to account for all of them at the same 
time in order to make sense of the situation. This unique feature inevitably creates a high demand on the cognitive resources [44] needed at a time for solving the simultaneous tasks. As a result, students fail to recognize multiple events and instead treat the situation as one indivisible whole. However, for sequential problems where multiple events occur consecutively, it is possible to consider one at a time, thereby circumventing the otherwise increased cognitive burden of handling multiple concepts all at once.

Our work provides useful implications for physics instruction. Since synthesis tasks differ from single-concept questions in demand for linking multiple events and concepts [45], it follows that students must comprehend diagrams and analyze situations qualitatively by connecting information from various sources, including problem text and diagram. As mentioned above, the act of making connections indicates that students are mentally processing and integrating information [30,39], a key factor in deep learning [40,41]. Synthesis problems demand in-depth conceptual insights [46] such that students must structure their mental representations of the situation and formulate appropriate strategies to allow for meaningful synthesis to happen. Overall, synthesis problems may encourage students' deep engagement with the tasks and result in enhanced cognitive activities. Therefore, they can be a useful format to help maximize problem solving as a learning tool for instruction.

Our study adds a new dimension to the literature on physics problem solving by exploring the influence of synthesis types on problem solvers' visual attention. Most of the earlier eye-tracking studies in PER have used singleconcept problems and focused predominantly on comparing between experts and novice students or between successful and unsuccessful problem solvers. However, they treated all physics problems as one unitary, single category. As shown in our study, problem solvers, be they successful or unsuccessful, can demonstrate different visual behaviors associated with the type of problems used. In that sense, our study contributes to the literature by highlighting the importance of taking this factor into consideration for future eye-tracking research in physics problem solving.

\section{ACKNOWLEDGMENTS}

This work is partially supported by Ohio State University College of Arts and Sciences-College of Education and Human Ecology Seed Grant, and by the National Science Foundation (Grant No. DRL-1252399).

\section{APPENDIX: AREAS OF INTERESTS FOR THE FOUR SYNTHESIS PROBLEMS}

The areas of interests (AOIs) for the four synthesis problems are shown in Fig. 5.

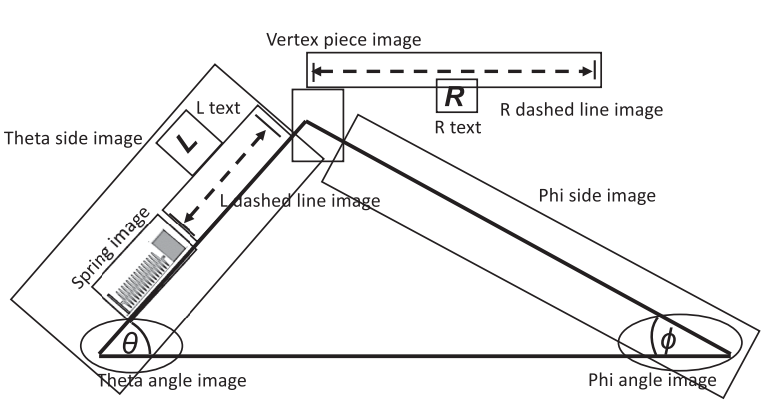

Q1

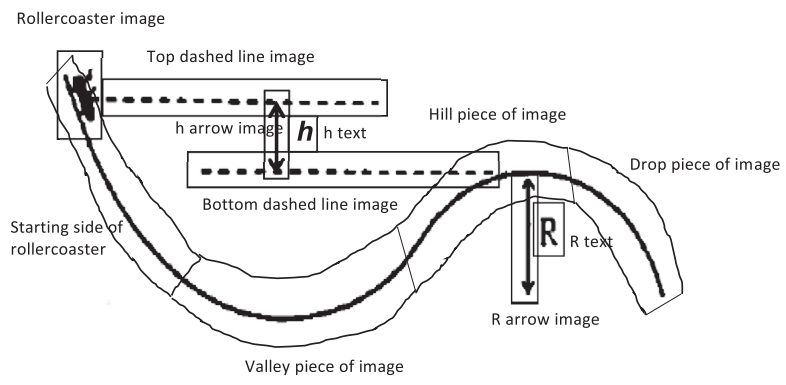

Q3

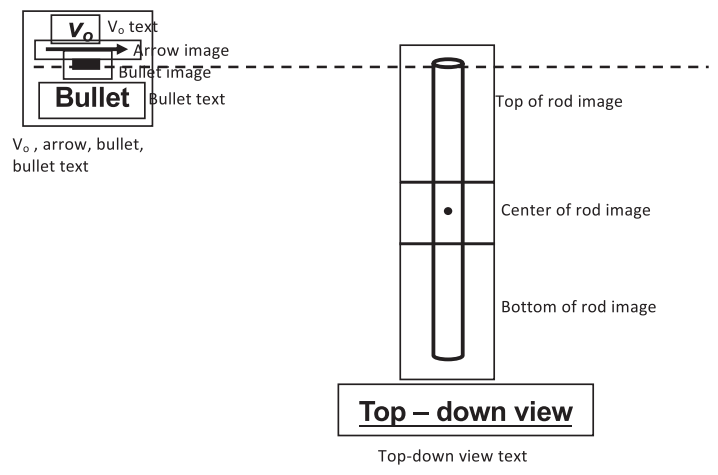

Q2

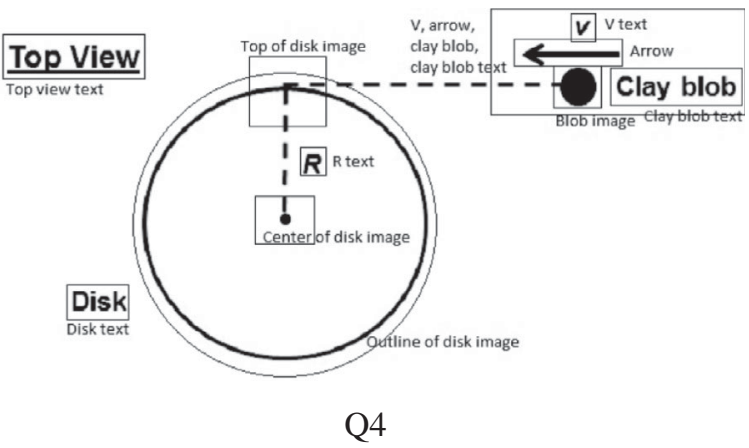

FIG. 5. AOIs for the sequential (Q1 and Q3) and simultaneous (Q2 and Q4) synthesis problems. 
[1] L. Hsu, E. Brewe, T. M. Foster, and K. A. Harper, Resource Letter RPS-1: Research in problem solving, Am. J. Phys. 72, 1147 (2004).

[2] J. L. Docktor and J. P. Mestre, Synthesis of disciplinebased education research in physics, Phys. Rev. Phys. Educ. Res. 10, 020119 (2014).

[3] P. B. Kohl and N. D. Finkelstein, Effect of representation on students solving physics problems: A fine-grained characterization, Phys. Rev. Phys. Educ. Res. 2, 010106 (2006).

[4] A. D. Smith, J. P. Mestre, and B. H. Ross, Eye-gaze patterns as students study worked-out examples in mechanics, Phys. Rev. Phys. Educ. Res. 6, 020118 (2010).

[5] A. Mason and C. Singh, Do advanced physics students learn from their mistakes without explicit intervention?, Am. J. Phys. 78, 760 (2010).

[6] D. P. Maloney, Getting started in PER, Reviews in PER Vol. 2 (American Association of Physics Teachers College Park, MD, 2011).

[7] B. Ibrahim and N. S. Rebello, Role of mental representations in problem solving: Students' approaches to nondirected tasks, Phys. Rev. Phys. Educ. Res. 9, 020106 (2013).

[8] B. Ibrahim and N. S. Rebello, Representational task formats and problem solving strategies in kinematics and work, Phys. Rev. Phys. Educ. Res. 8, 010126 (2012).

[9] C. A. Ogilvie, Changes in students' problem-solving strategies in a course that includes context-rich, multifaceted problems, Phys. Rev. Phys. Educ. Res. 5, 020102 (2009).

[10] L. Ding, N. Reay, A. Lee, and L. Bao, Exploring the role of conceptual scaffolding in solving synthesis problems, Phys. Rev. Phys. Educ. Res. 7, 020109 (2011).

[11] L. Ding, N. W. Reay, A. Lee, and L. Bao, Using conceptual scaffolding to foster effective problem solving, AIP Conf. Proc. 1179, 129 (2009).

[12] L. Ding, N. W. Reay, A. F. Heckler, and L. Bao, Sustained effects of solving conceptually-scaffolded synthesis problems, AIP Conf. Proc. 1289, 133 (2010).

[13] B. Ibrahim, L. Ding, A. F. Heckler, D. R. White, and R. Badeau, How students process equations in solving quantitative synthesis problems? Role of mathematical complexity in students' mathematical performance, Phys. Rev. Phys. Educ. Res. 13, 020120 (2017).

[14] B. Ibrahim, L. Ding, A. Heckler, D. White, and R. Badeau, Students' conceptual performance on synthesis physics problems with varying mathematical complexity, Phys. Rev. Phys. Educ. Res. 13, 010133 (2017).

[15] P. D. Antonenko, C. A. Ogilvie, D. S. Niderhauser, J. Jackman, P. Kumsaikaew, R. R. Marathe, and S. M. Ryan, Understanding student pathways in context-rich problems, Educ. Inf. Technol. 16, 323 (2011).

[16] S. Lin and C. Singh, Using isomorphic problems to learn introductory physics, Phys. Rev. Phys. Educ. Res. 7, 020104 (2011).

[17] R.Q. Xu, Internet computer coaches for introductory physics problem solving, Ph.D. dissertation, University of Minnesota, 2013 (to be published).

[18] A. M. Madsen, Studies of visual attention in physics problem solving, Ph.D. dissertation, Kansas State University, 2013 (to be published), https://krex.k-state.edu.
[19] K. Krejtz, A. T. Duchowski, I. Krejtz, A. Kopacz, and P. C. Wachtel, Gaze transitions when learning with multimedia, J. Eye Mov. Res. 9, 1 (2016).

[20] J. H. Lin and S. J. Lin, Tracking eye movements when solving geometry problems with handwriting devices, J. Eye Mov. Res. 7, 1 (2014).

[21] E. R. Grant and M. Spivey, Eye movements and problemsolving guiding attention guides thought, Psychol. Sci. 14, 462 (2003).

[22] M. J. Tsai, H. T. Hou, M. L. Lai, W. Y. Liu, and F. Y. Yang, Visual attention for solving multiple-choice science problem: An eye-tracking analysis, Comput. Educ. 58, 375 (2012).

[23] A. M. Madsen, A. M. Larson, L. C. Loschky, and N. S. Rebello, Differences in visual attention between those who correctly and incorrectly answer physics problems, Phys. Rev. Phys. Educ. Res. 8, 010122 (2012).

[24] S. S. M. Chanijani, S. S. Bukhari, P. Klein, J. Kuhn, M. Al-Naser, and A. Dengel, A study of representational competence in physics using mobile eye tracking systems, in Proceedings of the 18th International Conference on Human-Computer interaction with mobile devices and services (Association of Computing Machinery, 2016), pp. 1029-1032, https://doi.org/10.1145/2957265.2965012.

[25] M. Kekule, Students' approaches when dealing with kinematics graphs explored by eye tracking research method, in Proceedings of the Frontiers in Mathematics and Science Education Research Conference, FISER', edited by A. Bilsel and M. U. Garip (2014), 108-117, http://fiser.emu.edu.tr/2014/index.asp.

[26] H. Jing, L. Chen, Z. Fu, J. Fritchman, and L. Bao, Eyetracking of visual attention in web-based assessment using the Force Concept Inventory, Eur. J. Phys. 38, 1 (2017).

[27] A. Susac, A. Bubic, J. Kaponia, M. Planinic, and M. Palmovic, Eye movements reveal students' strategies in simple equation solving, Int. J. Sci. Math. Educ. 12, 555 (2014).

[28] F. J. Brigham, E. Zaimi, J. J. Matkins, J. Shields, J. McDonnough, and J. Jakubecy, The eyes may have it: Reconsidering eye-movement research and human cognition, in Advances in Learning and Behavioral Disabilities: Technological Applications, edited by T. E. Scruggs and M. A. Mastropieri (Elsevier Science New York, 2001), Vol. 15, pp. 39-59.

[29] D. D. Salvucci and J. R. Anderson, Automated eye-movement protocol analysis, Human-Computer Interactions 16, 39 (2001).

[30] C. I. Johnson and R. E. Mayer, An eye movement analysis of the spatial contiguity effect in multimedia learning, J. Exp. Psychol. Appl. 18, 178 (2012).

[31] M. A. Just and P. A. Carpenter, A theory of reading: From eye fixations to comprehension, Psychol. Rev. 87, 329 (1980).

[32] D. Irwin, Fixation location, and fixation durations as indices of cognitive processing, in The Interface of Language, Vision, and Action: Eye Movements and the Visual World, edited by J. M. Henderson and F. Ferreira (Psychology Press, New York, NY), pp. 105-133.

[33] S. Eivazi and R. Bednarik, Predicting problem-solving behavior and performance levels from visual attention data, 
in Proceedings of the Workshop on Eye Gaze in Intelligent Human Machine Interaction at IUI (2011), http://citeseerx .ist.psu.edu/viewdoc/download? doi=10.1.1.226.9272\&rep= rep1\&type=pdf.

[34] T. Zu, J. Hutson, L. Loschky, and N. S. Rebello, Using eye movements to measure intrinsic, extraneous, and germane load in a multimedia learning environment, J. Educ. Psychol. 112, 1338 (2019).

[35] D. Rosengrant, C. Thomson, and T. Mzoughi, Comparing experts and novices in solving electrical circuit problems with the help of eye-tracking, AIP Conf. Proc. 1179, 249 (2009).

[36] M. Hannus and J. Hyönä, Utilization of illustrations during learning of science textbook passages among low- and highability children, Contemp. Educ. Psychol. 24, 95 (1999).

[37] R. H. Tai, J. F. Loehr, and F. J. Brigham, An exploration of the use of eye-gaze tracking to study problem-solving on standardized science assessments, Int. J. Res. Meth. Educ. 29, 185 (2006).

[38] H. N. J. Ho, M. J. Tsai, C. Y. Wang, and C. C. C. Tsai, Prior knowledge, and online inquiry-based science reading: Evidence from eye tracking, Int. J. Sci. Math. Educ. 12, 525 (2014).

[39] R. E. Mayer, Multimedia Learning, 2nd ed. (Cambridge University Press, New York, 2009).

[40] L. Mason, P. Pluchino, and M. C. Tornatora, Using eyetracking technology as an indirect instruction tool to improve text and picture processing and learning. Br. J. Educ. Technol. 47, 1083 (2016).

[41] B. Park, A. Korbach, and R. Brünken, Do learner characteristics moderate the seductive-details-effect? A cognitiveload-study using eye tracking. J. Educ. Technol. Soc. 18, 24 (2015).

[42] R. Badeau, D. R. White, B. Ibrahim, L. Ding, and A. F. Heckler, What works with worked examples: Extending self-explanation and analogical comparison to synthesis problems, Phys. Rev. Phys. Educ. Res. 13, 020112 (2017).

[43] B. Ibrahim, L. Ding, D. R. White, R. Badeau, and A. F. Heckler, Synthesis problems: role of mathematical complexity in students' problem solving strategies, in Proceedings of the 2016 Physics Education Research Conference, Sacramento, CA, edited by D. L. Jones, L. Ding, and A. Traxler (AIP, New York, 2016), https://doi.org/10.1119/ perc.2016.pr.037.

[44] J. Sweller, Cognitive load during problem solving: Effects on learning, Cogn. Sci. 12, 257 (1988).

[45] A Lee, L. Ding, N. W. Reay, and L. Bao, Single-concept clicker question sequences, Phys. Teach. 49, 385 (2011).

[46] S. Ohlsson, Information-processing explanations of insight, and related phenomena, in Advances in the Psychology of Thinking, edited by T. Keane and K. J. Gilhooly (Harvester-Wheatsheaf, London, 1992). 